\title{
A comprehensive mathematical model for hybrid flexible flowshop lot streaming problem
}

\author{
Fantahun M. Defersha ${ }^{a}$
}

${ }^{a}$ School of Engineering, University of Guelph, 50 Stone Road East, Guelph, N1G 2W1,Canada

\begin{tabular}{l}
\hline A R T I C L E I N F O \\
\hline Article history: \\
Received 1 September 2010 \\
Received in revised form \\
15 October 2010 \\
Accepted 20 October 2010 \\
Available online 21 October 2010 \\
\hline Keywords: \\
Lot streaming \\
Scheduling \\
Hybrid flexible flowshop \\
Mathematical model
\end{tabular}

A B S T R A C T

\begin{abstract}
Lot streaming is a technique of splitting production lots into smaller sublots in a multi-stage manufacturing systems so that operations of a given lot can be overlapped. This technique can reduce manufacturing makespan and is an effective tool for time-based manufacturing strategy. Several research articles appeared in literature to solve this problem and most of these studies are limited to pure flowshop environments where there is only a single machine in each stage. On the other hand, because of the applicability of hybrid flowshops in different manufacturing settings, the scheduling of these types of shops is also extensively studied by several authors. However, the issue of lot streaming in hybrid flowshop environment is not well studied. In this paper, we aim to initiate research in bridging the gap between the research efforts in flowshop lot streaming and hybrid flowshop scheduling. We present a comprehensive mathematical model for scheduling flexible hybrid flowshop with lot streaming. Numerical example demonstrated that lot streaming can result in larger makespan reduction in hybrid flowshop where there is a limited research than in pure flowshop where research is abundant.
\end{abstract}

CC 2011 Growing Science Ltd. All rights reserved.

\section{Introduction}

Lot streaming is a technique to split a given job, each consisting of identical items, into sublots to allow overlapping of successive operations in multi-stage manufacturing systems thereby reducing production makespan. It is used to implement the time-based strategy in today's global competition (Chang \& Chiu, 2005). In fact, many world-class manufacturing companies (e.g., Dell and Toyota) have adopted this strategy to quickly produce and deliver goods to their customers (Blackburn, 1991; Bockerstette \& Shell, 1993). The concept of lot streaming was formally introduced in Reiter (1966) and its practice in flowshop is not new. Previous studies considering two-stage or special cases of three-stage flowshop lot streaming can be found in Potts and Baker (1989), Vickson and Alfredsson (1992), Trietsch and Baker (1993), Baker and Jia (1993), Glass et al. (1994), Baker (1995), Chen and Steiner (1996), Sen et al. (1998), and Sriskandarajah and Wagneur (1999). Lot streaming in flowshops with more than three stages has been studied recently by several researchers (see for

* Corresponding author. Tel: (519) 824-4120 Ext. 56512; Fax: (519) 836-0227

E-mail: fdefersh@uoguelph.ca (F.M. Defersha)

C 2011 Growing Science Ltd. All rights reserved. doi: $10.5267 /$ j.ijiec.2010.07.006 
example Kumar et al., 2000; Hall et al., 2003; Liu, 2003; Chiu et al., 2004; Bukchin \& Masin, 2004; Martin, 2009; Biskup \& Feldmann, 2006; Tseng \& Liao, 2008; Feldmann \& Biskup, 2008; Marimuthu et al., 2008).

A common feature of the above flowshop lot streaming studies is the assumption of single machine in each stage. However, the so called hybrid flowshops, where there are parallel machines at certain stages, are very common in industries. If one stage poses a bottleneck in the production process, managers may consider investing on additional machines for that stage. Moreover, hybrid flowshops have important applications in flexible manufacturing systems (FMS), manufacturing of electronics and furniture, process industries such as food processing, petrochemical and pharmaceutical industries (Xiao et al., 2000; Tang et al., 2005, 2006; Zandieh et al., 2006; Jungwattanakit et al., 2007). To this end, hybrid flowshop scheduling attracted much interest and proliffc scientiffc papers dealing with the diverse aspects of the problem appeared in literature (see for example BottaGenoulaz, 2000; Bertel \& Billaut, 2004; Oğuzc et al., 2004; Tang et al., 2005; Zhang et al., 2005; Tang et al., 2006; Ruiz \& Maroto, 2006; Zandieh et al., 2006; Ying \& Lin, 2006; Jungwattanakit et al., 2007; Janiak et al., 2007; Ruiz et al., 2008). Although this shows considerable research effort in hybrid flowshop scheduling, the issue of lot streaming in this type of shops has not gained as much attention as for pure flowshop scheduling. To the best of our knowledge the only paper that addressed lot streaming in hybrid flowshops is Zhang et al. (2005). However, this work is for a very special case where there are parallel machines only in the first stage and the number of stages is limited to two. Lot streaming in a more general hybrid flowshop where there can be parallel machines on any stage and the number of stages is not limited to two has been implicitly and partially addressed in Ruiz et al. (2008) through the concept of time-lag. Time-lag is defined as the difference between the completion time and the starting time of a job in two successively required stages, respectively. Ruiz et al. considered the overlapping of operations in successive stage by allowing negative time-lag. However, they assumed that this negative time-lag is a given data, not a decision variable to be optimized. Moreover, time-lag cannot fully represent lot streaming problem for the following reasons: It restricts the number of sublots of a given job only to two whereas theoretically the maximum number of sublots of jobs can be as large as the number of items in that job. Negative timelag between two successively required stages can assume values only within a certain range (see Ruiz et al., 2008). This in turn may not allow an optimal choice of sublot sizes. Time-lag cannot represent lot streaming when its value needs to be positive. A positive time-lag between successive operations of jobs is required when for example drying or cooling of products must occur before further operations can be carried out. Studies that allow the splitting of a job and the overlapping of its operations over the parallel machines in a given stage can be found in Bertel and Billaut (2004), Oğuzc et al. (2004), Oğuzc and Frinket (2005), and Ying and Lin (2006). The manufacturing systems considered in these studies are referred to as flowshop with multiprocessors (FSMP). These shops are special case of hybrid flowshops where the parallel machines are assumed to be identical. Though the studies in FSMP scheduling addressed the overlapping of operations over the parallel machines, such overlapping is not considered across stages. Thus, such studies may not be classified as lot streaming research as the very definition of lot streaming is to allow the overlapping of operations across stages.

The above literature review clearly indicates that there is a gap between the research efforts in flowshop lot streaming and hybrid flowshop scheduling. In this paper, we aim to initiate research in bridging this gap by developing a mathematical model for the general hybrid flowshop lot streaming problem. The proposed mathematical model incorporates lot streaming and other practical issues identified in Ruiz et al. (2008). These include (1) the existence of unrelated parallel machines, (2) the possibility of certain stages to be skipped by certain jobs, (3) sequence-dependent setup times, (4) anticipatory or non-anticipatory nature of setups, (5) release dates for machines, and (6) machine eligibility. A detailed discussion on these attributes can be found in Ruiz et al. (2008). Because of the first two attributes, the system considered in this study can be regarded as a complex generalized flowshop or hybrid flexible flowshop (HFFS). In Section 2 we present the proposed mixed integer 
linear programming model for HFFS lot streaming. Numerical example is given in Section 3 . Discussion and conclusion are in Section 4.

\section{Mathematical Formulation}

In this section, we present the problem description and the proposed MILP model for the type of flowshop lot streaming problem addressed in this paper. Most of the attributes of the proposed model were adopted from Ruiz et al. (2008) where the authors did not consider lot streaming.

\subsection{Problem Description and Assumptions}

Consider a flowshop consisting of several consecutive production stages to process several jobs. Each stage has a known number of unrelated parallel machines. The batch of each job is to be split into several unequal consistent sublots. The sublots are to be processed in the order of the stages and sublots of certain products may skip some stages. At a given stage, a sublot of a job can be assigned to one of the parallel machines eligible to process that particular job. For each job there is a sequence dependent setup time on each eligible machine and this setup may be anticipatory or non-anticipatory on different stages. Each machine can process at most one sublot at a time. Sublots of different products can be interleaved. The problem is to determine the size of each sublot of each job and the assignment and processing sequence of these sublots on each machine in each stage. The objective function is to minimize the completion time of the last sublot to be processed in the system.

\subsection{Notations}

In order to present a mathematical model for problem described above, we define several notations. These notations are explained here under.

\section{Indexes and Input Data:}

I Number of stages where stages are indexed by $i$ or $l=1,2, \cdots, I$

$M_{i} \quad$ Number of machines in stage $i$ where machines are indexed by $m$ or $k=1,2, \cdots, M_{i}$

$N \quad$ Number of jobs (products) where jobs are indexed by $n$ or $p=1,2, \cdots, N$

$J_{n} \quad$ Maximum number of sublots of job $n$ where sublots are indexed by $j$ or $s=1,2, \cdots, J_{n}$

$E_{n} \quad$ A set of pairs of stages $(l, i)$ for job $n$ constrained by precedence relations, i.e, the processing of job $n$ in stage $l$ is followed by its processing in stage $i$

$T_{n, m, i}$ Processing time for one unit of job $n$ on machine $m$ in stage $i$

$Q_{n} \quad$ Batch size of job $n$

$R_{m, i} \quad$ Maximum number of production runs of machine $m$ in stage $i$ where production runs are indexed by $r$ or $u=1,2, \cdots, R_{m, i}$

Theoretically $R_{m, i}$ is equal to the total number of sublots of all the jobs. However, because of the presence of parallel machines and skipping of stages by certain jobs, the number of sublots that will be actually processed on a particular machine at an optimal solution is likely lower than the total number of sublots. Hence, a reasonably lower value for $R_{m, i}$ has to be assumed in order to reduce the number of variable in the mathematical model.

$S_{m, i, n, p}$ Setup time on machine $m$ in stage $i$ for processing job $n$ following the processing of job $p$ on this machine; if $n=p$, the setup may be called minor setup 
$A_{n, i} \quad$ A binary data equal to 1 if setup of job $n$ in stage $i$ is attached setup (non-anticipatory), or 0 if this setup is detached setup (anticipatory)

$B_{n, i} \quad$ A binary data equal to 1 if job $n$ needs processing in stage $i$, otherwise 0

$D_{n, m . i}$ A binary data equal to 1 if job $n$ can be processed on machine $m$ in stage $i$, otherwise 0 ; $D_{n, m . i} \leq B_{n, i}$

$F_{m, i} \quad$ The release date of machine $m$ in stage $i$

$\Omega \quad$ Large positive number.

\section{Continuous Variables:}

$c_{j, n, i} \quad$ Completion time of the $j^{\text {th }}$ sublot of job $n$ in stage $i$

$\hat{c}_{r, m, j}$ Completion time of the $r^{\text {th }}$ run of machine $m$ in stage $i$

$\lambda_{j, n} \quad$ Size of the $j^{\text {th }}$ sublot of job $n$

$c_{\max }$ Makespan of the schedule

\section{Binary Variables:}

$x_{r, m, i, j, n} \quad$ Binary variable which takes the value 1 if the $r^{\text {th }}$ run on machine $m$ in stage $i$ is for the $j^{\text {th }}$ sublot of job $n, 0$ otherwise

$\gamma_{j, n} \quad$ Binary variable which takes the value 1 if sublot $j$ of job $n$ is non-zero $\left(\lambda_{j, n} \geq 1\right), 0$ otherwise

\subsection{MILP Model}

Using the notation given above, the objective function and the constraints of the proposed MILP mathematical model for the hybrid flexible flowshop lot streaming problem are presented below.

\section{Minimize:}

$Z=c_{\max }$

\section{subject to}

$$
\begin{aligned}
& \hat{c}_{r, m, i} \geq c_{j, n, i}+\Omega \cdot x_{r, m, i, j, n}-\Omega ; \forall(r, m, i, j, n) \\
& \hat{c}_{r, m, i} \leq c_{j, n, i}-\Omega \cdot x_{r, m, i, j, n}+\Omega ; \forall(r, m, i, j, n) \\
& \hat{c}_{1, m, i}-\lambda_{j, n} \cdot T_{n, m, i}-S_{m, i, n, 0}-\Omega \cdot x_{1, m, i, j, n}+\Omega \geq F_{m, i} ; \forall(m, i, j, n) \\
& \hat{c}_{r, m, i}-\lambda_{j, n} \cdot T_{n, m, i}-S_{m, i, n, p}-\Omega \cdot\left\{\left(\sum_{s=1}^{J_{p}} x_{r-1, m, i, s, p}\right)+x_{r, m, i, j, n}\right\}+2 \Omega \geq \hat{c}_{r-1, m, i} ; \\
& \forall(r, m, i, j, n, p) \mid r>1 \\
& \hat{c}_{1, m, i}-\lambda_{j, n} \cdot T_{n, m, i}-S_{m, i, n, 0} \cdot A_{n, i}-\Omega\left(x_{u, k, l, j, n}+x_{1, m, i, j, n}\right)+2 \Omega \geq \hat{c}_{u, k, l} ; \\
& \forall(u, k, m, l, i, j, n) \mid(l, i) \in E_{n}
\end{aligned}
$$




$$
\begin{aligned}
& \hat{c}_{r, m, i}-\lambda_{j, n} \cdot T_{n, m, i}-S_{m, i, n, \mathrm{p}} \cdot A_{n, i}-\Omega\left\{\left(\sum_{s=1}^{J_{p}} x_{r-1, m, i, s, p}\right)+x_{u, k, l, j, n}+x_{r, m, i, j, n}\right\}+3 \Omega \geq \\
& \hat{c}_{u, k, l} ; \forall(u, r, k, m, l, i, j, n, p) \mid(l, i) \in E_{n} \text { and } r>1 \\
& \sum_{n=1}^{N} \sum_{j=1}^{J_{n}} x_{r, m, i, j, n} \leq 1 ; \forall(r, m, i) \\
& \sum_{n=1}^{N} \sum_{j=1}^{J_{n}} x_{r+1, m, i, j, n} \leq \sum_{n=1}^{N} \sum_{j=1}^{J_{n}} x_{r, m, i, j, n} ; \forall(r, m, i) \mid r<R_{m, i} \\
& \sum_{j=1}^{J_{n}} \lambda_{j, n}=Q_{n} ; \forall n \\
& \lambda_{j, n} \leq \Omega \cdot \gamma_{j, n} ; \forall(j, n) \\
& \gamma_{j, n} \leq \lambda_{j, n} ; \forall(j, n) \\
& \sum_{m=1}^{M_{i}} \sum_{r=1}^{R_{m, i}} x_{r, m, i, j, n}=\gamma_{j, n} \cdot B_{n, i} ; \forall(i, j, n) \\
& x_{r, m, i, j, n} \leq D_{n, m, i} ; \forall(r, m, i, j, n) \\
& c_{m a x} \geq c_{j, n, i} ; \forall(j, n, i) \\
& x_{r, m, i, j, n} \text { and } \gamma_{j, n} \text { are binary }
\end{aligned}
$$

The objective function in Eq. (1) is to minimize the makespan of the schedule which is equal to the completion time of the last sublot processed in the system. The constraints in Eqs. (2) and (3) together state that the completion time of the $j^{\text {th }}$ sublot of job $n$ in stage $i$ is equal to the completion time of the $r^{\text {th }}$ run of machine $m$ in stage $i$ if this production run is assigned to that particular sublot. The starting time of the setup for the first run $(r=1)$ of machine $m$ in stage $i$ is given by $\hat{c}_{1, m, j}-\lambda_{j, n}$. $T_{n, m, i}-S_{m, i, n, 0}$ if the $j^{t h}$ sublot of job $n$ is assigned to this first run. This starting time cannot be less than the release date of the machine as enforced by the constraint in Eq. (4). The constraint in Eq. (5) is to enforce the requirement that the setup of any production run $r>1$ of a given machine cannot be started before the completion time of run $r-1$ of that machine. The constraint in Eq. (6) states that for any pair of stages $(l, i) \in E_{n}$, the setup or the actual processing of the first run on machine $m$ in stage $i$ may not be started before the completion time of run $u$ of machine $k$ in stage $l$, depending on whether the setup of product type $n$ in stage $i$ is non-anticipatory or anticipatory. This constraint is applied if run $u$ of machine $k$ in stage $l$ and that of the first run of machine $m$ in stage $i$ are both assigned to sublot $j$ of job $n$. The constraint in Eq. (7) is similar to that in Eq. (6) except Eq. (7) is for run $r>1$ of machine $m$ in stage $i$. In this case, the sequence dependent setup time has to be considered by taking into account the type of the job that was processed in run $r-1$ of machine $m$ in stage $i$. The constraint in Eq. (8) states that a production run $r$ of a particular machine $m$ at a particular stage $i$ can be assigned to at most on subplot. The constraint in Eq. (9) is to enforce the logic that a production run $r+1$ of a given machine can be assigned to a sublot if and only if run $r$ of that machine is already assigned to another sublot. The constraint in Eq. (10) enforces that the sum of the sizes of the sublots of a given job should be equal to the lot size of that particular job. Constraints in Eqs. (11) and (12) are to set the binary variable $\gamma_{j, n}$ to a value equal to 1 or zero depending on whether the size of $j^{\text {th }}$ sublot of job $n$ is positive or zero, respectively. If sublot $j$ of job $n$ is positive (i.e., $\gamma_{j, n}=1$ ) and it requires processing in stage $i$, then it should be assigned to one of the eligible machine in stage $i$. This is enforced by the constraints in Eqs. (13) and (14). Eq. (15) 
states that the makespan of the schedule, $c_{\max }$, is greater or equal to the completion time of any sublot on any stage. At optimality, $c_{\max }$ takes the value of the completion time of the last sublot to be processed in the system. Eq. (16) is the integrality requirement.

\section{Numerical Examples}

In flowshop lot streaming literature, most existing models and solution techniques are for pure flowshop systems. Research for hybrid flowshops has been limited, although lot streaming can be more effective in hybrid flowshops than in pure flowshops. In this numerical example, we demonstrate this fact by considering a small problem instance consisting of processing two jobs in three-stage pure and hybrid flowshops. The hybrid flowshop for this example consists of three identical machines in stage 1, two identical machines in stage 2, and other two identical machines in stage 3. The complete data for this example is given in Tables 1 and 2. For this small problem, the developed model was solved to optimality using CPLEX (version 11.2.0). The Gantt charts in Fig. 1a and Fig. 1-b show the schedules for the pure flowshop without and with lot streaming, respectively. The makespans for these two schedules are 1680 and 1152 minutes. The corresponding schedules for the hybrid flowshop are given in Fig. 2-a and Fig. 2-b with makespans of 1320 and 706 minutes, respectively. The percentages of makespan reduction due to lot streaming in pure and hybrid flowshop are $31 \%$ and $47 \%$, respectively. This result was obtained by setting the maximum possible number of sublots for each job to 3 . This problem was repeatedly solved by varying the maximum possible number of sublots for each job. The resulting makespans and the percentage makespan reductions are plotted in Fig. 3. As shown in Fig. 3-a, the makespans of the schedule in hybrid flowshop are shorter than those in pure flowshop because of the presence of parallel machines. A more interesting result is depicted in Fig. 3-b which shows the percentage of makespan reduction as the function of the maximum number of sublots for each job. The best makespan reduction in pure flowshop is $36 \%$ with maximum possible number of sublots being 6 . Increasing this number beyond 6 does not improve the makespan as the actual number of sublots does not increase due to the setup time for the sublots. The maximum makespan reduction in hybrid flowshop is $54 \%$. This is much better than the one achieved in the pure flowshop.

Table 1

Data set-1 for the example problem

\begin{tabular}{|c|c|c|c|c|c|c|c|c|c|c|c|}
\hline \multirow{3}{*}{$\begin{array}{l}\text { Stage } \\
i\end{array}$} & \multirow{3}{*}{$\begin{array}{l}\text { Machine } \\
m\end{array}$} & \multirow[b]{3}{*}{$F_{m, i}$} & \multirow[b]{3}{*}{$R_{m, i}$} & \multicolumn{8}{|c|}{ Job } \\
\hline & & & & \multicolumn{4}{|c|}{$n=1$} & \multicolumn{4}{|c|}{$n=2$} \\
\hline & & & & $B_{n, i}$ & $A_{n, i}$ & $D_{n, m . i}$ & $T_{n, m, i}$ & $B_{n, i}$ & $A_{n, i}$ & $D_{n, m, i}$ & $T_{n, m, i}$ \\
\hline \multirow[t]{4}{*}{1} & & & & 1 & 1 & & & 1 & 1 & & \\
\hline & 1 & 0 & & & & 1 & 4 & & & 1 & 6 \\
\hline & 2 & 0 & & & & 1 & 4 & & & 1 & 6 \\
\hline & 3 & 0 & & & & 1 & 4 & & & 1 & 6 \\
\hline \multirow[t]{3}{*}{2} & & & & 1 & 1 & & & 1 & 1 & & \\
\hline & 1 & 0 & 6 & & & 1 & 5 & & & 1 & 4 \\
\hline & 2 & 0 & 6 & & & 1 & 5 & & & 1 & 4 \\
\hline \multirow[t]{3}{*}{3} & & & & 1 & 1 & & & 1 & 1 & & \\
\hline & 1 & 0 & 6 & & & 1 & 6 & & & 1 & 5 \\
\hline & 2 & 0 & 6 & & & 1 & 6 & & & 1 & 5 \\
\hline \multicolumn{4}{|c|}{$J_{n}$} & \multicolumn{4}{|c|}{3} & \multicolumn{4}{|c|}{3} \\
\hline \multicolumn{4}{|c|}{$Q_{n}$} & \multicolumn{4}{|c|}{80} & \multicolumn{4}{|c|}{60} \\
\hline
\end{tabular}


Table 2

Data set-2 for the example problem

\begin{tabular}{llllllll}
\hline \multirow{2}{*}{$\begin{array}{l}\text { Stage } \\
i\end{array}$} & Machine & \multicolumn{3}{c}{$n=1$} & \multicolumn{3}{c}{$n=2$} \\
\cline { 2 - 7 } & $m$ & $S_{m, i, n, 0}$ & $S_{m, i, n, 1}$ & $S_{m, i, n, 2}$ & $S_{m, i, n, 0}$ & $S_{m, i, n, 1}$ & $S_{m, i, n, 2}$ \\
\hline 1 & 1 & 40 & 15 & 60 & 30 & 70 & 10 \\
& 2 & 40 & 15 & 60 & 30 & 70 & 10 \\
& 3 & 40 & 15 & 60 & 30 & 70 & 10 \\
2 & 1 & 30 & 10 & 45 & 20 & 50 & 16 \\
& 2 & 30 & 10 & 45 & 20 & 50 & 16 \\
3 & 1 & 50 & 10 & 50 & 40 & 60 & 10 \\
& 2 & 50 & 10 & 50 & 40 & 60 & 10 \\
\hline
\end{tabular}

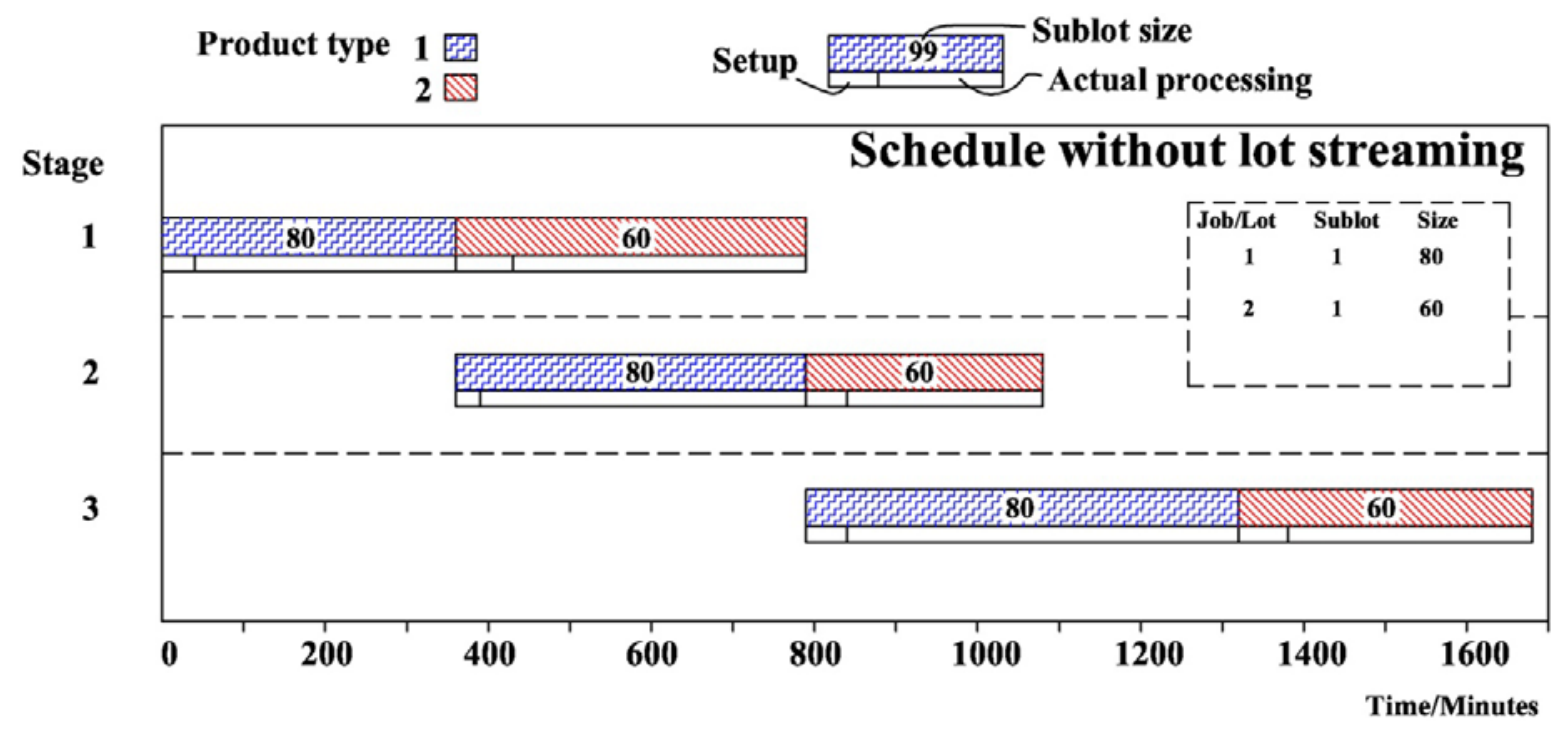

(a)

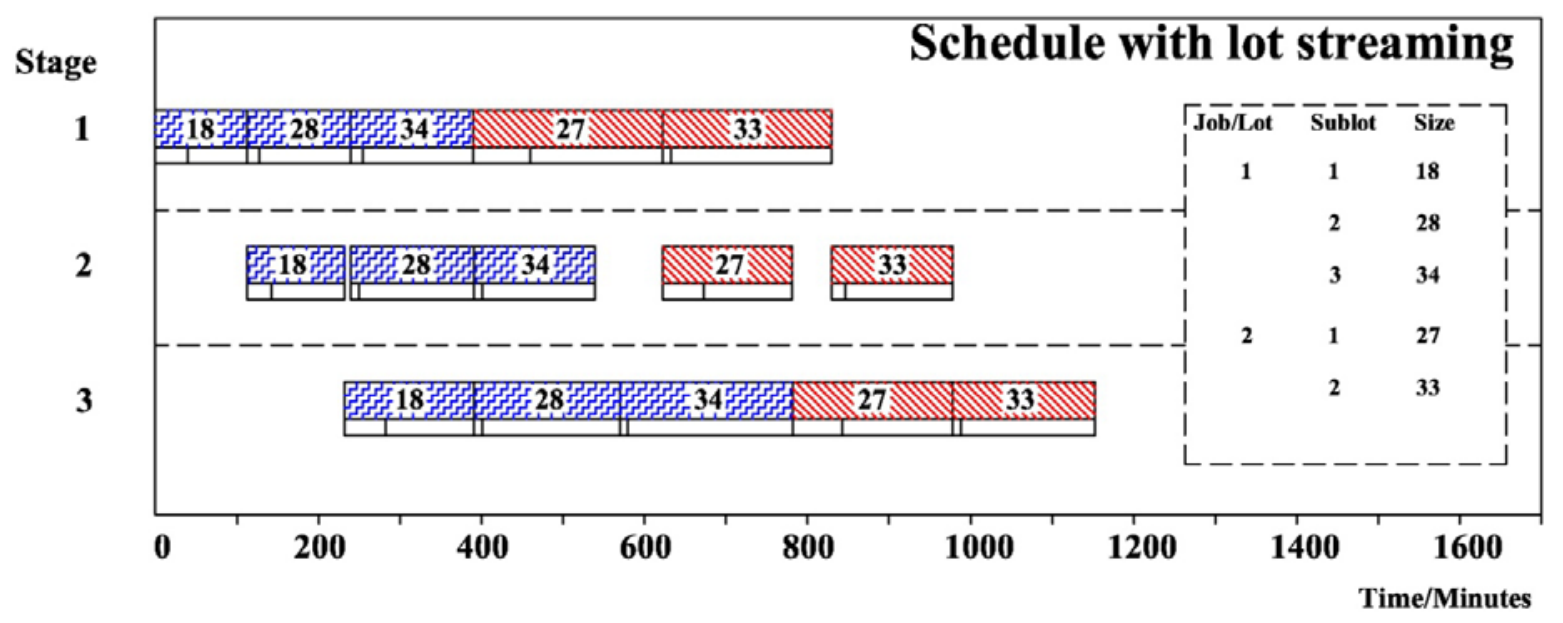

(b)

Fig. 1. Pure flowshop lot streaming for the example problem (a) without lot streaming and (b) with lot streaming 


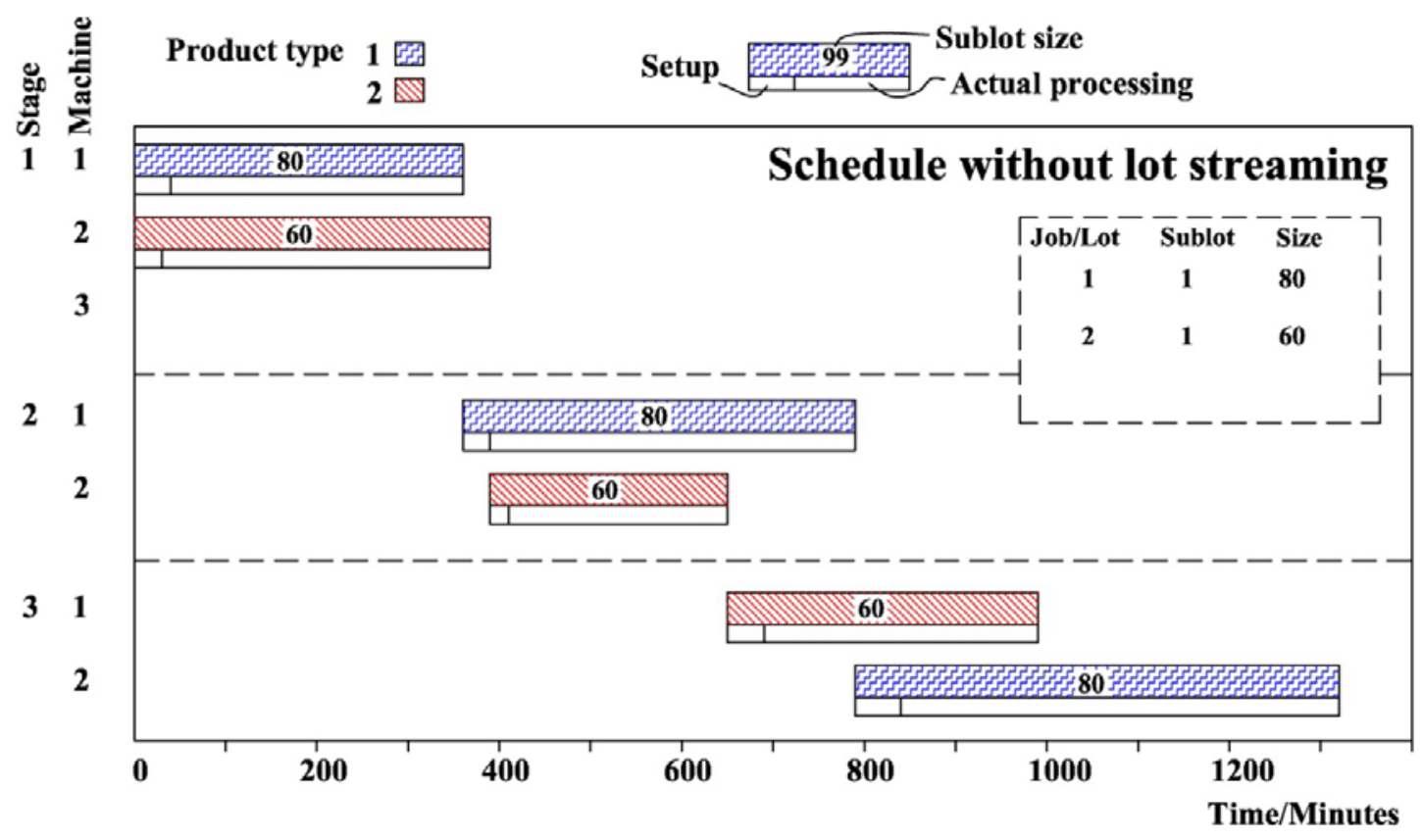

(a)

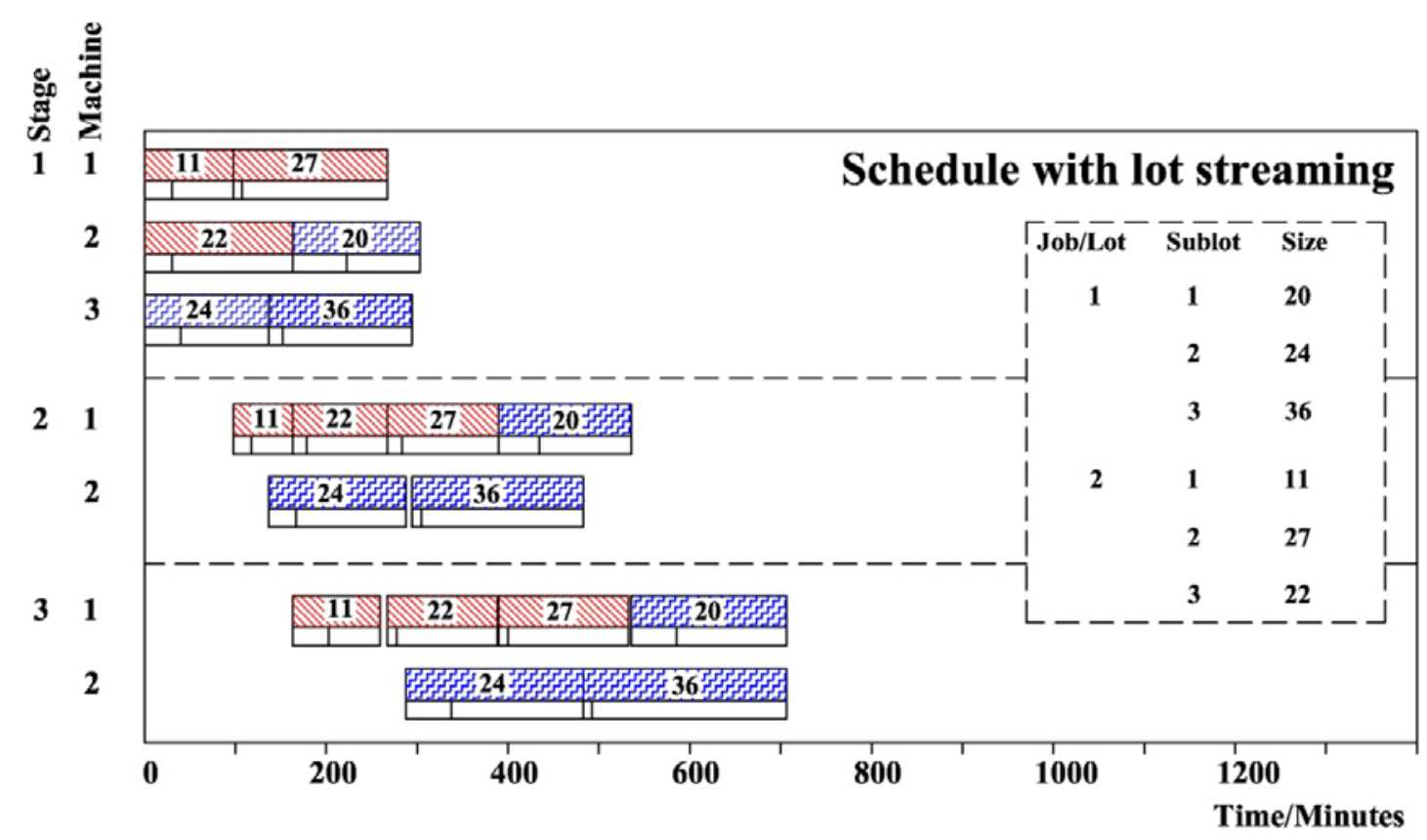

(b)

Fig. 2. Hybrid flowshop lot streaming for the example problem (a) without lot streaming and (b) with lot streaming.

We observed greater makespan reduction in hybrid flowshop which is basically because of the following two reasons: The first reason is that hybrid flowshops allow the overlapping of the operation of sublots of a given job not only across stages but also within stages as can be easily seen in Fig. 2-b. Another reason is that some stages in pure flowshop can easily pose bottleneck inhibiting further splitting of certain jobs. In order to elaborate this fact let us examine the schedule with lot streaming for the pure flowshop given in Fig. 1-b. This schedule is generated by assuming a maximum of 3 sublots for each job. However, an optimal solution was reached with three sublots for 
job 1 and with only two sublots for job 2. This is because, further splitting of job 2 will not improve the makespan as the third machine becomes a bottleneck. For instance, if the first sublot of job 2 (with size equal to 27) is split into two sublots of certain sizes, the completion time of these sublots can be shortened in the second stage. However, the completion time of these sublots in stage 3 cannot be shortened as this stage is occupied by sublot 3 of job 1 and cannot immediately process them. In the hybrid flowshop case, as can be seen in Fig. 2-b, all the three potential sublots of job 2 were generated to further reduce the makespan. Fig. 4 shows the number of sublots generated as a function of the maximum number of sublots in pure and hybrid flowshops. As can be seen in this figure, the actual number of sublots in the hybrid flowshop is larger to enable further makespan reduction.

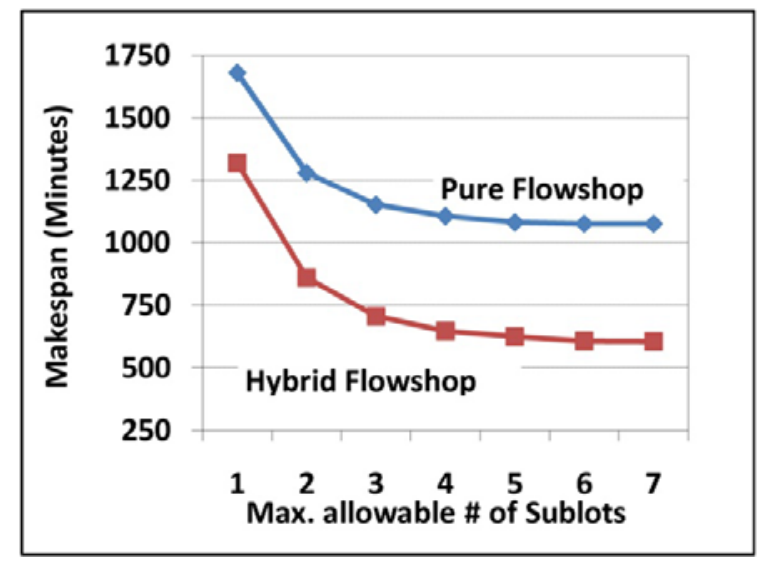

(a)

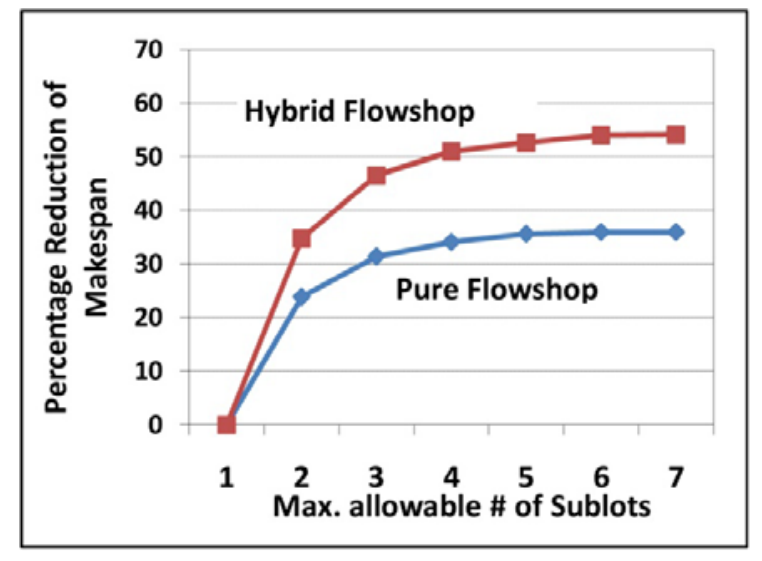

(b)

Fig. 3. (a) Makespan and (b) the percentage makespan reduction as functions of the maximum possible number of sublots of each job

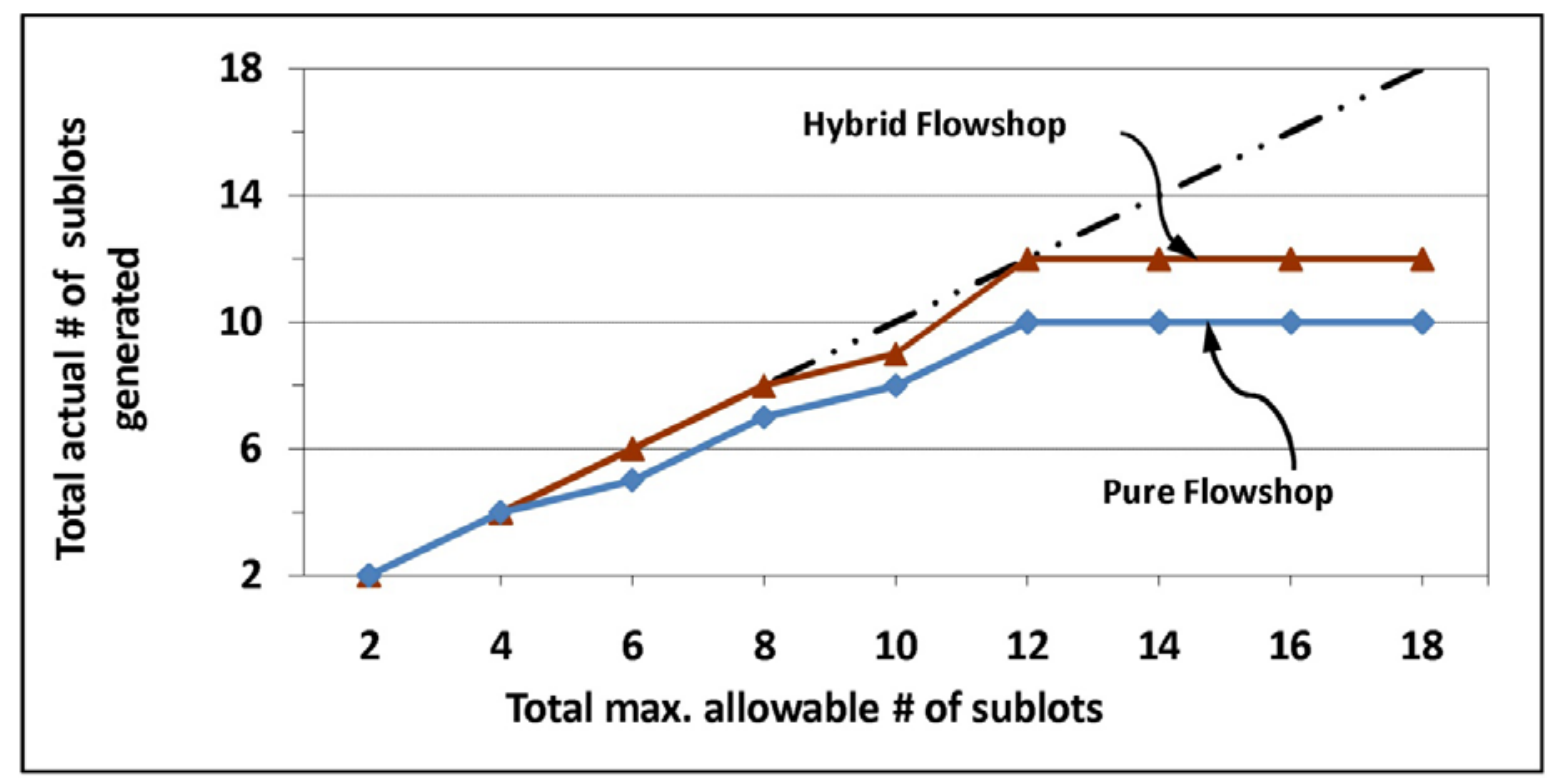

Fig. 4. Total actual number of sublots generated in pure and hybrid flowshops as the function of the maximum allowable number of sublots 


\section{Discussion and Conclusion}

This work was motivated by the gap we perceived in research efforts in pure flowshop lot streaming and hybrid flowshop scheduling. On one hand, because of a significant makespan reduction using lot streaming, there is a huge literature in pure flowshop lot streaming. On the other hand, because of the applicability of hybrid flowshops in different manufacturing settings, the scheduling of these types of shops is also extensively studied by several authors. However, lot streaming research in hybrid flowshops has been quite limited. In this paper, we aim to initiate research in bridging the gap between the research efforts in pure flowshop lot streaming and hybrid flowshop scheduling by presenting a comprehensive mathematical model for hybrid flowshop lot streaming. In addition to lot streaming, the mathematical model incorporates several other practical issues such as unrelated parallel machines, the possibility of certain jobs to skip certain stages, sequence-dependent setup times, anticipatory or non-anticipatory nature of setups, release dates for machines, and machine eligibility. The proposed model was solved to optimality for small problem size. The numerical example demonstrated that lot streaming can result in larger makespan reduction in hybrid flowshop where there is a limited research than in pure flowshop where research is abundant. In our future research, we plan to develop meta-heuristic algorithms to solve the proposed mathematical model efficiently. The model will also be further expanded to account for capacitated buffer size and capacitated material handling equipment, and inter-dependence of jobs because of assembly requirements. We also plan to develop models and solution procedures for lot streaming in nonconventional manufacturing system such as those based on cellular, fractal and distributed layouts where research is quite limited.

Acknowledgements: The author sincerely thanks the two anonymous referees for their thorough reviews of the early versions of this paper and their very valuable comments.

\section{References}

Baker, K. (1995). Lot streaming in the two-machine flow shop with setup times. Annals of Operations Research, 57, 1-11.

Baker, K. R. \& Jia, D., (1993). A comparative study of lot streaming procedures. OMEGA International Journal of Management Sciences, 21, 561-566.

Bertel, S. \& Billaut, J. C. (2004). A genetic algorithm for an industrial multiprocessor flow shop scheduling problem with recirculation. European Journal of Operational Research, 159, 651662.

Biskup, D. \& Feldmann, M. (2006). Lot streaming with varaibal sublots: an integer programming formulation. Journal of Operational Research Society, 57, 296-303.

Blackburn, J. (1991). Time-Based Competition. Business One Irwin, Burr Ridge, IL,

Bockerstette, J. \& Shell, R. (1993). Time Based Manufacturing. McGraw-Hill, New York,

Botta-Genoulaz, V. (2000). Hybrid flow shop scheduling with precedence constraints and time lags to minimize maximum lateness. International Journal of Production Economics, 64, 101-111.

Bukchin, J. \& Masin, M. (2004). Multi-objective lot splitting for a single product $\mathrm{m}$-machine flowshop line. IIE Transactions, 36, 191-202.

Chang, J. H. \& Chiu, H. N. (2005). A comprehensive review of lot streaming. International Journal of Production Research, 43, 1515-1536.

Chen, J. \& Steiner, G. (1996). Lot streaming with detached setups in three-machine flow shops. European Journal of Operational Research, 96, 591-611.

Chiu, H. N., Chang, J. H., \& Lee, C. H. (2004). Lot streaming models with a limited number of capacitated transporters in multistage batch production systems. Computers \& Operations Research, 31, 2003-2020.

Feldmann, M. \& Biskup, D. (2008). Lot streaming in a multiple product permutation flow shop with intermingling. International Journal of Production Research, 46, 197-216. 
Glass, C., JND, G., \& Potts, C. (1994). Lot streaming in three-stage process. European Journal of Operations Research, 75, 378-394.

Hall, N. G., Laporte, G., Selvarajah, E., \& Srikandarajah, C. (2003). Scheduling and lot streaming in flow shops with no-wait in process. Journal of Scheduling, 6, 339-354.

Janiak, A., Kozan, E., Lichtenstein, M., \& Oğuzc, C. (2007). Metaheuristic approaches to the hybrid flow shop scheduling problem with a cost-related criterion. International Journal of Production Economics, 105, 407-424.

Jungwattanakit, J., Reodecha, M., Chaovalitwongse, P., \& Werner, F. (2007). Algorithms for flexible flow shop problems with unrelated parallel machines, setup times, and dual criteria. International Journal of Advanced Manufacturing Technology, 37, 354-370.

Kumar, S., Bagchi, T., \& Sriskandarajah, C. (2000). Lot streaming and scheduling heuristics for mmachine no-wait flow shop. Computers and Industrial Engineering, 38, 149-172.

Liu, S. C. (2003). A heuristic method for discrete lot streaming with variable sublots in a flow shop. International Journal of Advanced Manufacturing Technology, 22, 662-668.

Marimuthu, S., Ponnambalam, S. G., \& Jawahar, A. N. (2008). Evolutionary algorithms for scheduling m-machine flow shop with lot streaming. Robotics and Computer-Integrated Manufacturing, 24, 125-139.

Martin, C. H., (2009). A hybrid genetic algorithm/mathematical programming approach to the multifamily flowshop scheduling problem with lot streaming. OMEGA International Journal of Management Sciences, 37, 126-137.

Oğuzc, C., Zinder, Y., Do, V. H., Janiak, A., \& Lichtenstein, M., (2004). Hybrid flow-shop scheduling problems with multiprocessor task systems. European Journal of Operational Research, 152, 115-131.

Oğuzc, C. \& Frinket, M. E. (2005). A genetic algorithm for hybrid flow-shop scheduling with multiprocessor tasks. Journal of Scheduling, 8, 323-351.

Potts, C. \& Baker, K. (1989). Flow shop scheduling with lot streaming. Operations Research Letter, 8, 297-303.

Reiter, S. (1966). A system for managing job shop production. Journal of Business, 34, 371-393.

Ruiz, R., Şerifoğlub, F. S., \& Urlings, T., (2008). Modeling realistic hybrid flexible flowshop scheduling problems. Computers \& Operations Research, 35, 1151-1175.

Ruiz, R. \& Maroto, C. (2006). A genetic algorithm for hybrid flowshops with sequence dependent setup times and machine eligibility. European Journal of Operational Research, 169, 781-800.

Sen, A., Topaloglu, E., \& Benli, O. S. (1998). Optimal streaming of a single job in a two-stage flow shop. European Journal of Operational Research, 110, 42-62.

Sriskandarajah, C. \& Wagneur, E. (1999). Lot streaming and scheduling multiple products in twomachine no-wait flow shop. IIE Transactions, 31, 695-707.

Tang, L., Liu, W., \& Liu, J., (2005). A neural network model and algorithm for the hybrid flow shop scheduling problem in a dynamic environment. Journal of Intelligent Manufacturing, 16, 361370.

Tang, L., Xuan, H., \& Liu, J. (2006). A new lagrangian relaxation algorithm for hybrid flowshop scheduling to minimize total weighted completion time. Computers \& Operations Research, 33, 3344-3359.

Trietsch, D. \& Baker, K., (1993). Basic techniques for lot streaming. Operations Research, 41, 10651076.

Tseng, C. T. \& Liao, C. J. (2008). A discrete particle swarm optimization for lot-streaming flowshop scheduling problem. European Journal of Operational Research, 191, 360-373.

Vickson, R. G. \& Alfredsson, B. E. (1992). Two and three machines flow shop scheduling problems with equal sized transfer batches. International Journal of Production Research, 30, 1551-1574.

Xiao, W., Hao, P., Zhang, S., \& Xu, X. (2000). Hybrid flow shop scheduling using genetic algorithms. Proceedings of the 3rd World Congress on Intelligent Control and Automation. Hefei, P.R. China, pp. 537-541. 
Ying, K.-C. \& Lin, S.-W. (2006). Multiprocessor task scheduling in multistage hybrid flow-shops: an ant colony system approach. International Journal of Production Research, 44, 3161-3177.

Zandieh, M., Fatemi Ghomi, S. M. T., \& Moattar Husseini, S. M. (2006). An immune algorithm approach to hybrid flow shops scheduling with sequence-dependent setup times. Applied Mathematics and Computation, 180, 111-127.

Zhang, W., Yin, C., Liu, J., \& Linn, R. J. (2005). Multi-job lot streaming to minimize the mean completion time in m-1 hybrid flowshops. International Journal of Production Economics, 96, 189-200. 